The University of San Francisco

USF Scholarship: a digital repository @ Gleeson Library | Geschke Center

$5-1-2000$

\title{
A Spectroscopic Redshift for the Cl 0024+16 Multiple Arc System: Implications for the Central Mass Distribution
}

Tom Broadhurst

Xiaosheng Huang

Brenda Frye

Richard Ellis

Follow this and additional works at: https://repository.usfca.edu/phys

Part of the Cosmology, Relativity, and Gravity Commons, and the Physics Commons 
Draft version July 16, 2018

Preprint typeset using LATEX style emulateapj v. 04/03/99

\title{
A SPECTROSCOPIC REDSHIFT FOR THE CL0024+16 MULTIPLE ARC SYSTEM: IMPLICATIONS FOR THE CENTRAL MASS DISTRIBUTION
}

\author{
Tom Broadhurst, Xiaosheng Huang, Brenda Frye, Richard Ellis \\ Department of Astronomy, University of California, Berkeley, CA 94720 \\ Institute of Astronomy, Madingley Road, Cambridge, CB3 0HA, UK
}

Draft version July 16, 2018

\begin{abstract}
We present a spectroscopic redshift of $z=1.675$ for the well-known multiply lensed system of arcs seen in the $z=0.39$ cluster Cl0024+16. In contrast to earlier work, we find that the lensed images are accurately reproduced by a projected mass distribution which traces the locations of the brightest cluster ellipticals, suggesting that the most significant minima of the cluster potential are not fully erased. The averaged mass profile is shallow and consistent with predictions of recent numerical simulations. The source redshift enables us to determine an enclosed cluster mass of $\mathrm{M}(<100 \mathrm{kpc} / \mathrm{h})=1.11 \pm 0.03 \times 10^{14} h^{-1} \mathrm{M}_{\odot}$ and a mass-to-light ratio of $\mathrm{M} / \mathrm{L}_{B}(<100 \mathrm{kpc} / \mathrm{h})=320 \mathrm{~h}\left(\mathrm{M} / \mathrm{L}_{B}\right) \odot$, after correction for passive stellar evolution. The arc spectrum contains many ionized absorption lines and closely resembles that of the local WolfRayet galaxy NGC4217. Our lens model predicts a high magnification $(\simeq 20)$ for each image and identifies a new pair of multiple images at a predicted redshift of $\mathrm{z}=1.3$.
\end{abstract}

Subject headings: cosmology: gravitational lensing — cosmology: observations - galaxies: clusters: individual $(\mathrm{Cl0024+16)}$ - galaxies: distances and redshifts

\section{INTRODUCTION}

The $z=0.39$ rich cluster Cl0024+16 (Zwicky 1959) displays one of the finest examples of gravitational lensing. Four clearly related images are identified around the tangential critical curve in HST WF/PC1 data (e.g. Smail etal. 1996). A further radially directed image of the same source was later found in a refurbished HST WFPC-2 image by Colley et al. (1996). These arcs have been used by Colley et al. (1996) to construct an image of the source and by Tyson et al. (1998) to examine details of the mass distribution.

For many years the redshift of this lensed source has eluded identification despite long exposures on large telescopes. The blue colour and lack of optical emission lines suggests a redshift $1<\mathrm{z}<2$ (Mellier et al. 1991). The importance of the redshift for lensing studies lies primarily in measuring the central mass and mass-to-light ratio of the lensing cluster, and in the case of Cl0024+16 these quantities can be measured particularly accurately for this cluster because of the symmetric arrangement of the images. The results will depend on the source redshift through its effect on the ratio of lens to source separations, $d_{L S} / d_{S}$. For a nearby lens this ratio saturates very quickly with increasing source redshift, but for a more distant lens like Cl0024+16 there is a larger range of $d_{L S} / d_{S}$ and hence a larger uncertainty in the mass, depending on the source redshift.

\section{OBSERVATIONS}

The HST imaging data used here is that obtained by Colley et al. (1996). The archival data was first reduced for the purposes of selecting lensed targets for mulislit spectroscopy. Briefly, the images comprise seven exposures totaling 8400 s in the $\mathrm{F} 450 \mathrm{~W}$ band and six exposures totaling 6600s in the F814W band. Images were aligned to the nearest integer pixel and cosmic-ray rejected to obtain an average flux. With multislits on LRIS at the $10 \mathrm{~m}$ Keck II telescope a $15^{\prime \prime}$ slit was centered on a high surface-brightness feature of the largest, and hence most magnified arc (upper end of arc A, see Fig 2a) to maximize the detection of spectral features. The total exposure time was 80 minutes in 0.8 arcsec seeing, using the 300 line grating blazed at $5000 \AA$, providing a useful wavelength range of $4500 \AA-9500 \AA$. The resulting spectrum is shown in Fig 1, revealing many absorption lines, with a redshift of $\mathrm{z}=1.675$. The spectrum, observed at an average resolution of $6 \AA$ in the restframe matches closely that of the nearby starburst galaxy NGC4214 (Leitherer et al. 1996), showing a similar continuum slope and common absorption lines of SiII $1527 \AA$, CIV $1550 \AA$, FeII $1608 \AA, 2344 \AA, 2382 \AA$, $2600 \AA$, AlII $1671 \AA$, AlIII $1859 \AA$. We also detect a 
appeared initially to be related to the lensed arcs but is difficult to reproduce in the lens model (Fig 2b).

\section{MODELLING THE CLUSTER LENS}

Lens models for Cl0024+16 have generally improved with higher quality imaging. Using ground based data, Kassiola et al. (1994) and Wallington et al. (1995) reproduced fits to the close triplet of arcs (A,B,C of Figure 2) but considered arc D an unlikely counter image. Subsequently HST images revealed that A,B,C and D are morphologically similar in detail (Smail et al. 1996) and that a further radially directed arc in the cluster center, E, is another complete image of the same source (Colley et al. 1996). Recently a 512 parameter fit to the resolved imaging data for the arc system has been presented by Tyson et al. (1998). This solution required the inclusion of a number of small dark deflecting 'mascons' around each of the images to offset the symmetry of a dominant central potential (see Fig 2 of Tyson et al. 1998).

To investigate the uncertainties in mass we revisit the mass model using a simple approach which we nonetheless find sufficient to reproduce the basic properties of the image configuration. We start by assigning profiles to the brightest cluster members using the form advocated by NFW (Navarro, Frenk and White 1995), which has a characteristic scale, $r_{s}$, and dimensionless normalization relative to the cosmological critical density, $\delta_{c}=\rho_{s} / \rho_{\text {crit }}$, and allows a wide range of mass concentrations. Integrating the mass along a column, $\mathrm{z}$, where $r^{2}=\left(\xi_{r} r_{s}\right)^{2}+z^{2}$ gives

$$
\mathrm{M}\left(\xi_{\mathrm{r}}\right)=\rho_{\mathrm{s}} \mathrm{r}_{\mathrm{s}}^{3}\left(\xi_{\mathrm{r}}\right) \int_{\mathrm{o}}^{\xi_{\mathrm{r}}} \mathrm{d}^{2} \xi_{\mathrm{r}} \int_{-\infty}^{\infty} \frac{1}{\left(\mathrm{r} / \mathrm{r}_{\mathrm{s}}\right)\left(1+\mathrm{r} / \mathrm{r}_{\mathrm{s}}\right)^{2}} \frac{\mathrm{dz}}{\mathrm{r}_{\mathrm{s}}}
$$

resulting in a deflection angle $\alpha(\theta)=\frac{4 G M(<\theta)}{c^{2} \theta d_{L}} \frac{d_{L S}}{d_{S}}$ in the image plane at position $\vec{\theta}=\vec{\xi}_{r} r_{s} / d_{L}$.

Only the brightest 8 cluster members need be included to generate an accurate fit (all cD galaxies, see Fig 2a) with $r_{s}$ and $\delta_{c}$ unconstrained, corresponding to a simple vector addition of deflection fields. Inclusion of many fainter members produces noise as the fit rapidly becomes unconstrained for the small number of independent constraints (5 images). The fit is achieved with the "downhill simplex" algorithm (Press et al. ) by minimizing the difference between the model predicted locations of the three obvious features (HII regions) common to the 5 main images of the source i.e.

$$
\chi^{2}=\sum_{k} \sum_{i, j, i>j}\left(\left(\vec{\theta}_{i, k}-\vec{\alpha}\left(\vec{\theta}_{i, k}\right)\right)-\left(\vec{\theta}_{j, k}-\vec{\alpha}\left(\vec{\theta}_{j, k}\right)\right)\right)^{2}
$$

a sum over all $\mathrm{k}$ points of all images.

The projected (2-D) mass density contours shown in fig 2 are modulated by the cluster members despite the projection. The fit favours an overall shallow profile centered on the central tight clump of luminous galaxies (fig 2a,b). The two outer ellipticals are seen to be responsible for the largest departure from symmetry generating the triple images $\mathrm{A}, \mathrm{B}, \& \mathrm{C}$. The solution although good is not of course unique, since a set of discrete profiles generates a fairly smooth potential (see Fig 2b) and for this reason we do not need to introduce additional unknown parameters to describe a diffuse component. We can convert the the azimuthally-averaged projected model slope of $\theta^{-0.55}$ in the vicinity of $100 \mathrm{kpc} / \mathrm{h}$ for comparison with an NFW profile. The conversion to a real space slope is $\gamma=-1-2 \frac{\xi_{r}}{1+\xi_{r}}$, or $\gamma=-1.26$ at the critical radius. This is very close to the NFW expectation for massive clusters which have a predicted slope of $\sim-1.3$ at $\sim 100 \mathrm{kpc} / \mathrm{h}$ (NFW, Ghingna et al. 1998) corresponding to a combination of $r_{s} \sim 400 \mathrm{kpc} / \mathrm{h}$ and $\delta_{c} \sim 8000$.

\section{MASS/LIGHT RATIOS}

The rest-frame luminosities of the cluster galaxies are converted from data numbers to an ST-magnitude using header information. The instrumental colours of the bright ellipticals are very similar with a mean of $V_{450 W}-I_{814 W}=1.62(2.818$ in the AB system or 3.334 normalised to Vega) which in the Johnson system $B-I=3.7$ (Holzman et al. 1996) and corresponds well to a passively evolved old stellar population which at the observed redshift $z=0.39$, with $z_{f}=3$ and $\tau=.01$ (Bruzual \& Charlot 1995) predicts colours of $B-I=3.825$ (3.727) for $\Omega=0.1(\Omega=1)$ and $\mathrm{h}=0.5$ (solar metallicity). Correction to the present requires removal of $0 .^{m} 49-0 .^{m} 52$ of passive evolution.

The arcs define a convenient radial aperture for comparing mass and light with a radius of 30.5 arcsec radius intersecting the four bright tangential images. Using the observed lens and source redshifts, we normalise the mass distribution by the ratio $d_{L S} / d_{S}=0.61$ (virtually independent of curvature). Integrating over our model mass distribution yields $M(\theta)=1.28 \times 10^{14} h^{-1} M_{\odot}$ for $\Omega=0.1$, corresponding to a metric radius of $\sim 106 \mathrm{kpc} / \mathrm{h}$ at the lens. Note $\Omega$ enters only via the angular-diameter distance of the lens, so that for $\Omega=1$ the mass is lower by $9 \%$ for the same angular aperture. The model mass is close to the Einstein mass of a symmetric lens, $M\left(\theta<30.5^{\prime \prime}\right)_{\text {crit }}=1.33 \times 10^{14} M_{\odot}, \quad(\Omega=0.1$ and independent of profile) as expected given the near circular arrangement of the images about the cluster which means we have confidence in the mass to an accuracy of less than $\sim 2 \%$.

The integrated I-band light in this aperture is $I_{814 W}^{S T}=16.2$ or $L_{B}(\theta)=3.94 \times 10^{11} h^{-2} L_{\odot}(\Omega=$ 0.1 ) after subtracting passive evolution above and hence, the central a mass-light ratio is, $M / L_{B}(\theta)=$ 
$324 h\left(M / L_{B}\right) \odot$ at $z=0$ (using $M_{B \odot}=5.48$ ). This is slightly higher than other lensing clusters (Kneib etal. 1996, Natarajan et al. 1998), but note that neglecting evolution reduces $M / L_{B}$ by $50 \%$, and should be allowed for in accurate comparisons between clusters. This value is short of that necessary for closure estimated from local redshift surveys in the same passband estimated requires $\left(M / L_{B}\right)_{\text {crit }}=$ $1500_{-400}^{+700} h(M / L)_{B \odot} \quad$ (Efstathiou, Ellis \& Peterson 1988) corresponding to a larger volume and a later mean galaxy type.

The true size of the source galaxy after subtraction of the deflection field of image radius, is found to be $r \sim 0.25^{\prime \prime}, \sim 20$ times fainter than the tangential images, with an unlensed luminosity $M_{B}=$ $-20.85 \pm 0.7-5 \log h$ and an apparent magnitude of $I \sim 24.8$, typical of what may be expected for a galaxy at the measured redshift (see Figure 18 of Bouwens, Broadhurst \& Silk 1998).

\section{NEW MULTIPLE IMAGES}

New multiply lensed images may be sought with our lens model to check and improve upon the model. The unknown redshift introduces an additional free parameter from the distance dependence in the bend angle. Hence to search for new multiple images we need only take the deflection field $\vec{\alpha}(\vec{\theta})$ defined for the five arc system at $z_{1}=1.67$ with $\left(d_{L S} / d_{S}\right)_{z_{1}}$ and multiply by a scalar, $f=\left(d_{L S} / d_{S}\right)_{z_{2}} /\left(d_{L S} / d_{S}\right)_{z_{1}}$, mimicking the effect of a change in the source redshift.

In practice it is difficult to securely identify new images, mainly because galaxies are similarly blue and numerous so that a unique identification based on only 2 passbands is difficult. Furthermore arcs which are obviously radial or tangential lie close or straddle the critical curves and therefore much more magnified than their counterimages which consequently may be too faint to detect. Furthermore, for a given mass distribution a source must lie above some minimum redshift to generate multiple images, corresponding to $\mathrm{z}>1.0$ on average for sources within the einstein ring of Cl0024+16. Here we claim a secure identification of one new pair of arcs as shown on figure 2. The relative deflection is $90 \%$ of that of the main arcs corresponding to a predicted redshift of $\mathrm{z}=1.31, \mathrm{z}=1.34, \mathrm{z}=1.33$ for $\Omega=0.05, \Omega=1, \Omega+\Lambda=0.3+$ 0.7. In principle then a sufficiently accurate lens model can produce a geometric constraint on the cos- mological curvature, however many more multiple images of higher redshift sources need to be identified to make this practical.

\section{DISCUSSION AND CONCLUSIONS}

It is clear from the above modeling that some degree of mass substructure is required in Cl0024+16, contrary to the conclusions of Tyson et al. (1998). The large mass-to-light ratio we assign to the location of the luminous ellipticals is far in excess of isolated elliptical galaxies, meaning these galaxies simply trace well local minima of a general potential. This finding is consistent with the substructure apparent in all carefully studied lensing clusters, notably, A2218, A370, AC114, A2390, Cl0939+47 (Kneib etal. 1996, Smail etal. 1996, Abdelsalam etal. 1998, Pierre etal. 1996, Natarajan etal. 1997, Seitz et al. 1996). It is not clear if this level of substructure is in excess of N-body predictions which, as Ghigna et al. (1997) point out are certainly underestimates within the central $50 \mathrm{Kpc} / \mathrm{h}$ where the problem of "overmerging" is still significant despite their superior dynamic range.

Our azimuthally averaged mass profile is shallow, in good agreement with recent high-resolution CDM Nbody simulations, corresponding to an NFW profile with $r_{s} \sim 400 \mathrm{kpc} / \mathrm{h}$ which is four times greater than the observed einstein ring radius and leads to large central image magnifications. Although our model does not explicitly incorporate a separate diffuse component it can be seen in Fig 2, that the sum of the eight profiles forms a generally smooth mass distribution illustrating the degeneracy of this sort of modeling and hence the redundancy of a separate provision for diffuse matter.

We have successfully used our lens model to find new multiply lensed galaxies. It is clear that with more color information further systems will be distinguished in an iterative procedure where the mass model is successively refined with the incorporation of the new images. Relative distance predictions can be made this way for comparison with measured redshifts allowing, in principle, a geometric measure of the cosmological curvature.

We thank Rychard Bouwens and Ben Moore for useful conversations. TJB acknowledges NASA grant AR07522.01-96A.

\section{REFERENCES}

Abdelsalam,H.M., Saha,P.,Williams, L.L.R., 1998, AJ,116,1541

Bouwens, R., Broadhurst, T., \& Silk, J., 1998, ApJ, 506,557

Bruzual, G., \& Charlot, S., 1993, ApJ., 405,538

Colley, W.N..Tyson, J.A., Turner, E.L., 1996, ApJ 461, L83

Efstathiou, G., Ellis, R.S., Peterson, B.A., 1988 MNRAS 221,233
Ghigna, S., Moore, B., Governato, F., Lake, G., Quinn, T., Steidel, J., 1998, MNRAS, 300,146

Holtzman, J., Burrows, C., Casertano, S., Jeff, H., Trauger, J., Watson, A.., Worthey, G.. 1995, PASP, 107,1065

Kassiola, Å., Kovner, I. \& Blandford, R. D. 1992, ApJ, 396, 10 


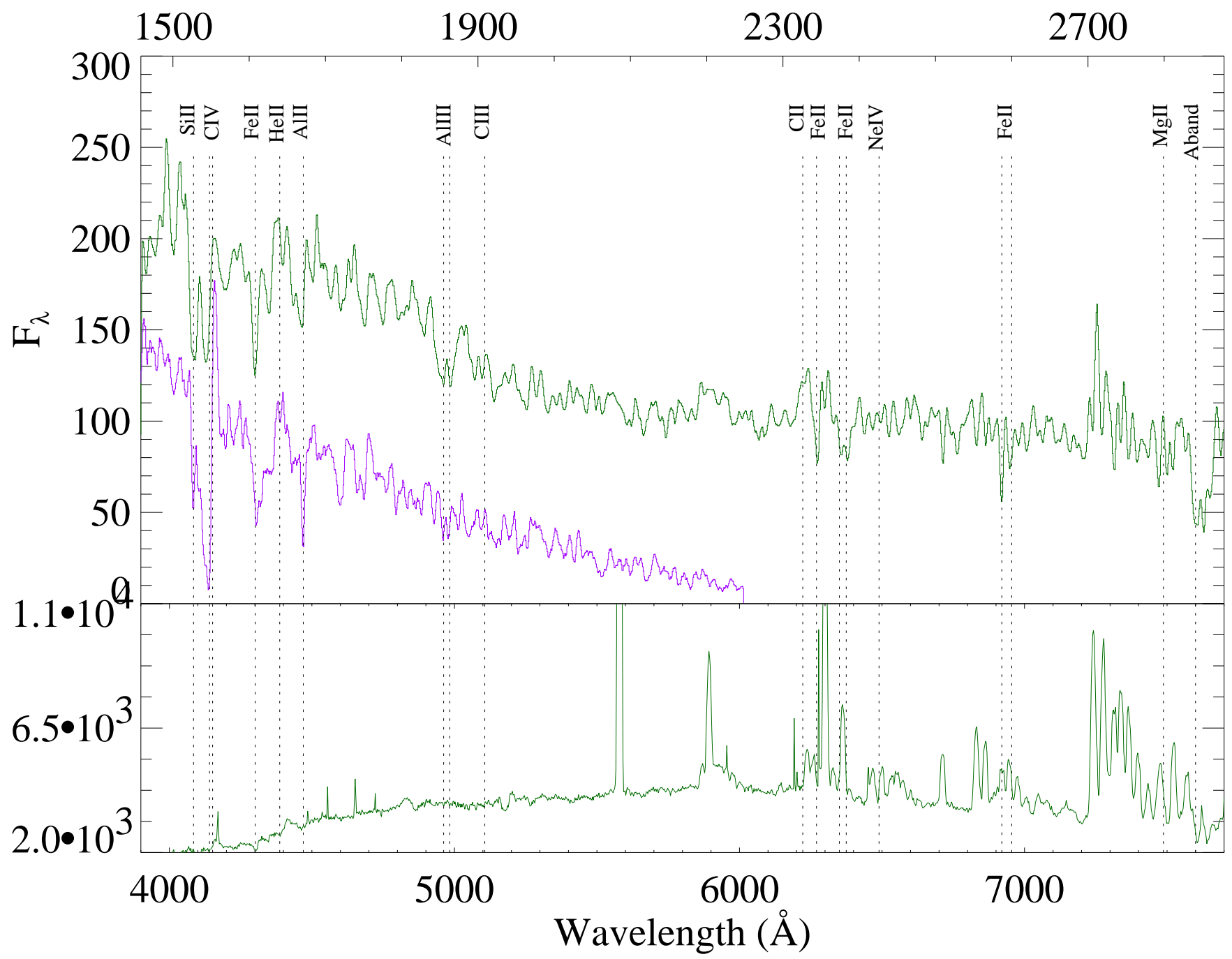

FIG. 1. - The upper curve is fluxed spectrum ofi the upper HII region of arc C (fig 2b). Many weak absorption lines are visible yielding an unambiguous redshift of $\mathrm{z}=1.675$. Note the similarity with the local "Wolf-Rayet" galaxy (lower curve) NGC4214 (Leitherer 1996). The sky spectrum is also shown, in the lower panel

Kneib, J.P.,Ellis, R.S.,Samil, I., Couch, W.J., \& Sharples, R.M. 1996, ApJ. 471643

Leitherer,C.L.,Vacca, W.D., Conti, P.S., Carmelle, R., Sargent, W.L.W., 1996, ApJ, 465,717

Mellier, Y., Fort, B.,Soucail, G., Mathez, G., Cailloux, M., 1991ApJ, 380, 334

Natarajan, P., Kneib, J-P., Smail, I.S., Ellis, R.S., 1998, ApJ, 499,600

Pierre, M., Le Borgne, J.F., Soucail, G., Kneib, J.P., 1996, A\&A, 311,413
Smail, I., Dressler, A; Kneib, JP; Ellis, R.S., Couch W.J., Sharples, R.M.,Oemler, A.. 1996, ApJ, 469,508

Seitz, C, Kneib, J.-P., Schneider, P. \& Seitz, S. 1996, A\&A,314,707

Tyson, J.A., Kochanski, G.P., Dell'Antonio I.P., 1998, ApJ 498,107

Wallington, S., Kochanek, C.S., Koo, D.C., 1995, ApJ, 441,58

Zwicky,F. 1959, Handbuch der Physik,53,390 

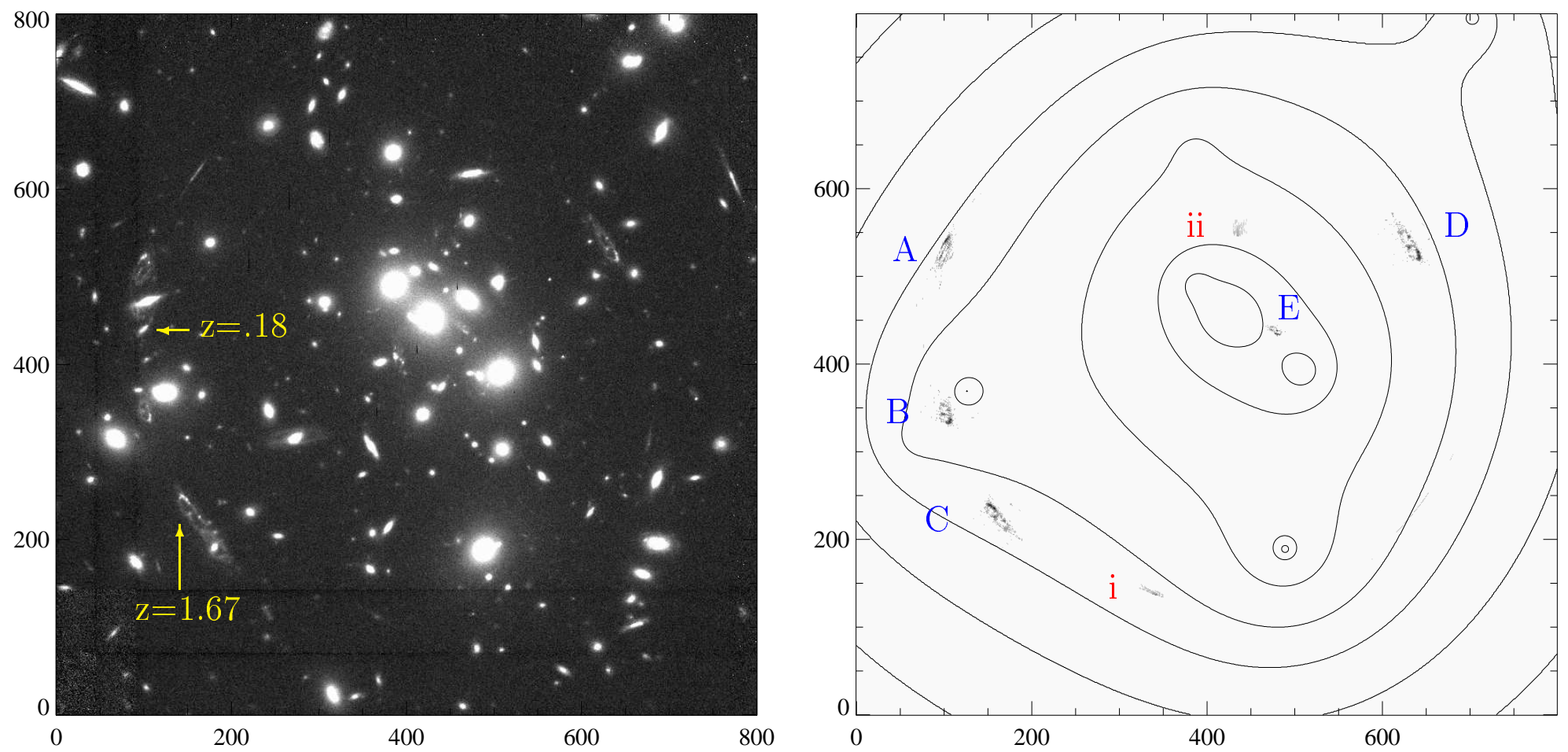

Fig. 2. - The central region of the summed B+I band images is shown on the left for comparison with the model on the right. Note the good agreement with the 5 main images (A-E). These may be improved further by hand, incorporating masses local to each image, but will not alter the conclusion that the central mass distribution traces well the location of the brightest ellipticals. A new multiple image pair is identified by the model (i,ii) at a predicted redshift of $z \simeq 1.3$. The contours represent surface mass density from $(0.6-1.2) \Sigma_{\text {crit }}$, separated by 0.1 . 